\title{
IMPORTÂNCIA DA DETECÇÃO DE PATOLOGIAS DO DESENVOLVIMENTO NA ANÓXIA NEONATAL E DA INTERVENÇÃO FISIOTERÁPICA
}

\author{
CLAUDIA MORAES TREVISAN*, ANGELA TERESINHA ZUCHETTO** ELENITA COSTA BEBER ***, \\ IENNETE BERNADETE SCHUCH ${ }^{* * *}$, MARIA DE FATIMA SARTORI ***, \\ VERA APARECIDA ROSSATTO***, ANA LUCIA RICK ${ }^{* * *}$, SANDRA BELMONTE***
}

\begin{abstract}
RESUMO - O objetivo deste estudo foi verificar o desenvolvimento neuropsicomotor e a necessidade de estimulação nos 27 casos com recuperação parcial e diagnóstico de anóxia neonatal atendidos no ano de 1988 na UTI de Recém-Nascidos (RN) do Hospital Universitário de Santa Maria-RS. Foi relacionado o diagnóstico de patologia neurológica e a indicação fisioterápica no momento da alta hospitalar. Os RN foram avaliados segundo abordagem neuroevolutiva em visitas domiciliares a 10 casos. Os resultados revelaram a necessidade de intervenção fisioterápica nos casos de anóxia neonatal.
\end{abstract}

PALAVRAS-CHAVES: fisioterapia, anoxia cerebral, patologias do desenvolvimento.

\section{Importance of development disorders detection in newborn anoxy and physiotherapy intervention}

SUMMARY - The purpose of this study was to verify the neuropsychomotor development and the necessity of stimulation, correlating the neurological pathology diagnosis to the physioterapeutic indication at the moment of hospital discharging. Cases studied were 27 patients with partial recovery and newborn anoxy diagnosis assisted in 1988 at the Newborn Intensive Care Unit of the University Hospital in Santa Maria-RS. The newborns were evaluated according to neuroevolutive approach during domiciliary visits to 10 patients. The results showed the necessity of physiotherapeutic intervation on cases of newborn anoxy.

KEY WORDS: physiotherapy, cerebral anoxy, development disorders.

Anóxia neonatal é fator determinante da hipoxia, na qual o fluxo sanguíneo é lento para garantir a oxigenação, podendo resultar danos em orgãos como rins, coração e cérebro ${ }^{14}$. Diversos estudos mostraram que anóxia é a causa associada com maior frequência ao diagnóstico de patologia neurol6gica ${ }^{1,20,23,24}$. Ela pode ocorrer mesmo que o parto tenha sido normal ${ }^{7}$.Os avanços na área do desenvolvimento infantil preconizam a identificação precoce do transtorno cerebral e motor, assim como de outros retardos infantis. Estes têm sido transferidos cada vez mais no curso dos últimos anos para os três primeiros meses de vida do lactente, pelo reconhecimento de que devido a sua grande plasticidade nesta fase, o cérebro infantil melhor se adapta a manipulações externas ${ }^{12,13,17}$. A evolução do cérebro infantil durante esse período se acompanha da capacidade, quase predizível, de

Estudo realizado no Hospital Universitário de Santa Maria ( HUSM) Universidade Federal de Santa Maria-RS (UFSM): *Professora Assistente do Departamento de Fisioterapia e Reabilitação, UFSM, Mestranda em Crescimento e Desenvolvimento Humano; **Professora Assistente do Departamento de Pratica Esportiva, UFSC, Mestre em Ciência do Movimento, ${ }^{* * *}$ Acadêmicos do Curso de Fisioterapia, UFSM. Aceite: 26-maio1994.

Prof " Claudia Moraes Trevisan - Departamento de Fisioterapia e Reabilitação, Centro de Ciências da Saúde, UFSM - 97119-900 Santa Maria RS - Brasil. 
aprendizagem relacionada com a maturação e com a estimulação ambiental ${ }^{17}$. É por isso que, nessa fase precoce, as medidas fisioterápicas têm efeito mais complexo e global do que se consegue apos a posterior diferenciação cerebral. Estudos fundamentais sobre o problema do reconhecimento precoce e do tratamento precoce da paralisia cerebral desenvolveram-se de 1927 a $1972^{13}$. Foram os estudos de Bobath (1952-1975) ${ }^{4,5}$ que melhor conscientizaram os pediatras de que patologias do desenvolvimento se tratavam de problema particularmente dos primeiros anos de vida, com possibilidades terapêuticas Foi sistematizado o conhecimento da paralisia cerebral, possibilitando seu reconhecimento precoce e proporcionando apoio terapêutico também precoce. A intervenção na patologia do movimento sugerida por Bobath ${ }^{5}$ baseia-se na abordagem neuroevolutiva em que é verificada, através da avaliação, a interferência da lesão na maturação normal do encéfalo, e, assim, possibilitando estabelecer diretrizes para a abordagem terapêutica ${ }^{4}$.

Baseando-se nessas considerações, o objetivo deste estudo foi verificar, nos 27 casos de recuperação parcial e diagnóstico de anóxia neonatal atendidos em 1988 na UTI de Recém-Nascidos do HUSM, seu desenvolvimento neuropsicomotor, relacionando-o ao diagnóstico de patologia neurológica e à indicação fisioterápica no momento de alta hospitalar.

\section{MATERIAL E MÉTODOS}

Foi enviada correspondência para os responsáveis pelas 27 crianças com diagnóstico de anóxia neonatal com recuperação parcial atendidos na UTI de Récem-Nascidos do HUSM no ano de 1988, decorridos 12 meses do diagnóstico, para avaliação neuroevolutiva no Serviço de Fisioterapia do HUSM. Como não se obteve resposta, foram realizadas visitas domiciliares a 10 casos. As demais crianças não foram encontradas.

Esses 10 casos foram avaliados segundo a abordagem neuroevolutiva Bobath, pela qual anormalidades nos padrões motores podem ser detectadas ${ }^{4}$.

\section{RESULTADOS}

Os resultados são apresentados nas Tabelas 1, 2, 3 e 4.

\section{COMENTÁRIOS}

Pode-se observar na Tabela 1 que 20\% dos casos apresentaram avaliação neuroevolutiva alterada. As avaliações neuroevolutivas ${ }^{1,4,5,20,23}$ são capazes de permitir predizer as alterações neurologicas posteriores. As avaliações em recém-nascidos (RN) permitem identificar distúrbios do comportamento ${ }^{6}$. Isto encontra suporte nos dados de diversos autores que mostram ser a avaliação a única forma de diagnosticar a necessidade de estimulação 1,20,22,23.

Conforme a Tabela 2, a necessidade de estimulação foi detectada em $20 \%$ dos casos. Brandão², salienta a necessidade de encaminhar para tratamento o mais breve possível, crianças que apresentam diagnóstico de patologia que possa vir a comprometer seu desenvolvimento. Segundo esse mesmo autor, a estimulação atua através da atividade passível de ser produzida na criança, estando destinada a impulsionar funções pré existentes, para as quais o estímulo só será eficaz e adequado quando estiver integrado a todo o sistema funcional e estrutural da criança, valorizando a relação afetiva da criança e seu ambiente. Para Finger ${ }^{12}$, logo após a verificação de alguma anormalidade na criança,

Tabela 1. Avaliação neuroevolutiva.

\begin{tabular}{lcc}
\hline \multicolumn{1}{c}{ Variável } & $\mathrm{n}$ & $\%$ \\
\hline \hline Normal & 8 & 80 \\
Alterado & 2 & 20 \\
Total & 10 & 100 \\
\hline
\end{tabular}

Tabela 2. Necessidade de estimulação.

\begin{tabular}{lcc}
\hline \multicolumn{1}{c}{ Variável } & $\mathrm{n}$ & $\%$ \\
\hline \hline Sim & 2 & 20 \\
Não & 8 & 80 \\
Total & 10 & 100 \\
\hline
\end{tabular}


Tabela 3. Diagnóstico de patologia neurologica.

\begin{tabular}{lcc}
\hline \multicolumn{1}{c}{ Variável } & $\mathrm{n}$ & $\%$ \\
\hline \hline Sem informações & 0 & 0 \\
Sim & 1 & 3,7 \\
Não & 26 & 96,3 \\
Total & 27 & 100 \\
\hline
\end{tabular}

Tabela 4. Indicação fisioterápica.

\begin{tabular}{lcc}
\hline Recuperação parcial & $\mathrm{n}$ & $\%$ \\
\hline \hline Sem informações & 25 & 93 \\
Sim & 2 & 7 \\
Não & 0 & 0 \\
Total & 27 & 100 \\
\hline
\end{tabular}

mesmo uma pequena cianose, deve ser indicado o encaminhamento para a estimulação, pois este trabalho irá inibir, em cerca de $90 \%$ ou mais, a instalação de um quadro patológico.

Conforme se mostra na Tabela 3,96,3\% dos casos não apresentaram patologias neurológicas; apenas 3,7\% apresentaram diagnóstico deste tipo de patologia. Segundo Fischer e Paton (apud Ramos e Vaz. ${ }^{21}$ ), a lesão cerebral ocorre mais facilmente quando a anóxia prolongada é maior que os mecanismos de compensação orgânica. Mulligan e col. (apud Ramos e Vaz ${ }^{21}$ ), em 65 RN com anóxia que sobreviveram e foram acompanhados desde o nascimento até os 5 anos de idade, observaram incidência de sequelas neurológicas graves em 48,5\%. Rotta e col..$^{23}$ comentam que, de $90 \mathrm{RN}$ de alto risco com anóxia estudados, 20 apresentavam exame neurológico (EN) alterado e 70, normal. Acompanharam 75 desses RN até os 7 anos, observando que $70 \%$ apresentavam EN evolutivo alterado, concluindo que o EN patológico do RN foi capaz de predizer em $80 \%$ as alterações neurológicas posteriormente constatadas. Gonzales e col. ${ }^{16}$ comentam a incidência de anóxia em 14,6x1000 RN vivos. Entre estes RN a mortalidade foi $1,25 \times 1000 \mathrm{RN}$, a letalidade $8,5 \%$ e a porcentagem de sequelas $3,6 \%$. Esses autores salientam a importância da anóxia no prognóstico de crianças pré-termo. Low e col. ${ }^{20}$, estudando a relação entre hipóxia perinatal e encefalopatia do RN, observaram que esta última foi encontrada em $30 \%$ de $303 \mathrm{RN}$ de alto risco (pré-termo e a termo). Robertson e Finner ${ }^{22}$, em estudo sobre RN a termo com encefalopatia hipóxico-isquêmica em seguimento por 3,5 anos, verificaram que, do total dos $167 \mathrm{RN}$ a termo, 66 apresentavam anóxia moderada ao nascimento e, destes, $21,3 \%$ apresentaram deficiências. Silva e col ${ }^{24}$ monstraram, em acompanhamento de 39 RN com anóxia neonatal, que na evolução $29,5 \%$ apresentaram retardo no desenvolvimento neurológico. Berroteran e col. ${ }^{1}$, acompanhando por controle periódico 105 crianças com anóxia neonatal, observaram que: $75,23 \%$ evoluíram satisfatoriamente e $19,04 \%$ apresentaram problemas neurológicos. Entre estes, 2 com paralisia cerebral, 2 com retardo mental severo, 4 com crises convulsivas e 1 com epilepsia e retardo mental. Os estudos analisados mostram que a anóxia é a causa associada com maior frequência ao diagnóstico de patologia neurológica.

Conforme a Tabela 4, em $93 \%$ não havia informações sobre indicação fisioterápica e somente $7 \%$ receberam indicação para que ela fosse feita. Para Leitão ${ }^{19}$, a mielinização das fibras do sistema nervoso central começa em torno do $4^{\circ}$ mês de vida fetal e os principais tratos estão mielinizados em torno do $2^{\circ}$ ano de vida. A vulnerabilidade do cérebro do RN é grande, o que é devido a seu alto metabolismo e a suas reservas energéticas baixas. Na gênese das lesões cerebrais a hipoxemia isquêmica determinada pela anóxia constitui processo crítico, evolutivo e agressivo.

A Fisioterapia deve ser introduzida precocemente e de forma orientada para o desenvolvimento e deve ser adaptada ao distúrbio sensóriomotor do indivíduo ${ }^{13}$. Esta intervenção terapêutica pode ser propícia ao desenvolvimento de funções psicomotoras. Este estímulo às funções, segundo Diament ${ }^{9}$ concorre para o desenvolvimento mais rápido da mielinização. Para Brandão ${ }^{2}$, a intervenção precoce é atributo da área da saúde em razão de tratar com patologias do movimento, não podendo ser desenvolvida de forma adequada com pessoal sem formação específica. 
Em conclusão, os resultados obtidos sugerem que: (a) crianças com anóxia neonatal podem apresentar comprometimento no desenvolvimento neuropsicomotor; (b) há necessidade do exame neuroevolutivo em crianças com esse diagnóstico; (c) é imprescindível o acompanhamento com enfoque multidisciplinar; (d) há necessidade de maior sensibilização de fisioterapeutas e profissionais da equipe multidisciplinar atuantes nessa área. Com base nas possibilidades terapêuticas que vêm sendo aprimoradas nos últimos anos, Kong ${ }^{18} \mathrm{e}$ Bobath ${ }^{4}$, sugerem que no tratamento de crianças com atraso no desenvolvimento devemos abandonar o comportamento expectante: observam-se peculiaridades inicialmente discretas do desenvolvimento do lactente, que vão dar origem a retardo inequívoco da criança.

\section{REFERÊNCIAS}

1. Berroteran O, Bello A, Salas L, Finol R. Seguimiento de niños egresados de la seccion de observacion pedriátrica de la maternidade Concepcion Palacios. Rev Obst Ginecol (Venezuela) 1981, 4: 237-242.

2. Brandão P. Presença indispensável. Rev APAE 1985, 1:30.

3. Brandão P. Estimulação precoce . Rev APAE 1985,1:30.

4. Bobath B, Bobath K. Desenvolvimento motor nos diferentes tipos de paralisia cerebral. São Paulo: Manole, 1978.

5. Bobath K. Uma base neurofisiológica para o tratamento da paralisia cerebral. São Paulo: Manole, 1984.

6. Brunialdi R. Avaliação motora em bebês : tabela de Dubowitzin. X Congresso Nacional da Associação Brasileira de Paralisia Cerebral, São Paulo, 1989, p 49.

7. Cambier J, Masson M, Dehein H. Manual de neurologia. Rio de Janeiro: Masson Brasil, 1980.

8. Colby L, Kisner C. Exercícios terapêuticos.São Paulo: Manole, 1989.

9. Diament AJ. Bases do desenvolvimento neurológico. Arq Neuropsiquiatr 1978, 36:285-302.

10. Downie P. Neurologia para fisioterapeutas.São Paulo: Panamericana .1987.

11. Duarte JP. Socorro obstetras ajudem-nos. Bol Clínica de Paralisia Cerebral Dr. Júlio Pinto Duarte 1972, 1:5.

12. Finger JA. Terapia ocupacional. São Paulo: Sarvier. 1986.

13. Flehming I. Desenvolvimento normal e seus desvios no lactente. Rio de Janeiro: Atheneu, 1987.

14. Ganong WF. Fisiologia médica.São Paulo: Atheneu, 1985.

15. Glock R. A fisioterapia na otimização do processo educativo de criança normais. Anais VII Congresso Brasileiro de Fisioterapia, 1985,p 96.

16. Gonzales HC, Davila GO, Lavieta G. P, Jimenes C. H, Schaff L. G, Moldonado JS. Aspectos epidemiológicos de la encefelopatía hipóxica-isquémica. Bol Méd Hosp Inf. Mex. 1985, 42:541-546.

17. Kogler E. Intervenção precoce. Fisioterapia em Movimento 1991, 4: 108-111.

18. Kong E. Very early treatment of cerebral palsy. Child Nerol 1966, 8:206-209.

19. Leitão A. Paralisia cerebral, diagnóstico, terapia e reabilitação.Rio de Janeiro: Atheneu, 1983.

20. Low JA., Galbraith RS, Muir DW, Killem HL, Pater EA, Karchmar EJ. The relationship between perinatal hipoxia and newborn encephalopathy. Am J Obst Gynecol 1985, 152: 256-260.

21. Ramos JLA, Vaz FAC. Pediatria neonatal.São Paulo: Sarvier,1978.

22. Robertson C, Finner. N. Term infants with hypoxic-ischemic encephalopathy: outcome at 35 years. Dev Med Child Neurol 1985, 27:473-484.

23. Rotta NT, Winckler MIB, Lyra A., Ohlweiler L, Lago IS. Alterações neurológicas a longo prazo em recém nascidos de alto risco. X Congresso Brasileiro de Neurologia e Psiquiatria Infantil. São Paulo, 1989, p 33.

24. Silva EA., Pessoto MA., Ribeiro NVLM. Acompanhamento de 39 casos de recém-nascidos com anóxia neonatal grave. X Congresso de Neurologia e Psiquiatria Infantil. São Paulo, 1989, p 32. 